PROCEEDINGS OF THE

AMERICAN MATHEMATICAL SOCIETY

Volume 127, Number 7, Pages 2109-2115

S 0002-9939(99)04729-2

Article electronically published on March 3, 1999

\title{
NONLINEAR CARLEMAN OPERATORS ON BANACH LATTICES
}

\author{
WILLIAM FELDMAN
}

(Communicated by Palle E. T. Jorgensen)

\begin{abstract}
An operator, not necessarily linear, will be called a Carleman operator if the image of the positive elements in the unit ball are bounded in the universal completion of the range space. For certain Banach lattices, a class of (not necessarily linear) Carleman operators is characterized in terms of an integral representation and in a more general setting as operators satisfying a pointwise finiteness condition. These operators though not linear are orthogonally additive and monotone.
\end{abstract}

The notion of a Carleman operator from a normed space into a Riesz space was formulated by Grobler and van Eldik [4]. Namely, a linear map $T$ from $E$ into $F$ is a Carleman operator if the image of the unit ball under $T$ is order bounded in the universal completion of $F$. For a general operator $T$, we define $T$ to be a Carleman operator if the image of the positive elements in the unit ball are order bounded in the universal completion of $F$. In this note, a class of operators that are orthogonally additive, monotone, and satisfy a condition with respect to scalar multiplication is considered. We provide a characterization of a Carleman operator among the continuous operators in this class as a type of integral operator (Theorem 1) and in Theorem 2, we provide a characterization of a Carleman operator for operators satisfying a translation property. In the absence of a measure, Theorem 3 provides a characterization of Carleman operators in terms of the finiteness condition. For linear Carleman operators, characterizations have been provided by the author in [3]. Orthogonally additive operators have been considered in a variety of settings, including several papers by Mazón and De León analyzing order bounded orthogonally additive operators (e.g., see [2], and [5]).

\section{PRELIMINARIES}

We will assume that the Banach lattice $E$ is representable in the sense of Schaefer [6], that is, there exists a locally compact topological space $X$ with the property that $E$ is Riesz isomorphic to an ideal in $C^{\infty}(X)$, all the continuous extended realvalued functions (range $[-\infty, \infty]$ ), each finite on a dense subset of $X$. We will study those Banach lattices with quasi-interior points and thus representable on a compact space $X$. Specifically, $e$ in $E$ is a quasi-interior point if the order ideal generated by $e$ is dense in $E$. The ideal generated by $e$ is then identified with $C(X)$, the continuous real-valued functions on a compact space $X$. Here, the space

Received by the editors December 9, 1996 and, in revised form, October 16, 1997.

1991 Mathematics Subject Classification. Primary 46B42, 47H07.

(C)1999 American Mathematical Society 
$X$ can be viewed as the lattice homomorphisms on the order ideal generated by $e$ together with the product topology. An element $f$ in the ideal is now identified with a continuous function $\hat{f}$ on $X$ by defining $\hat{f}(x)=x(f)$ for each homomorphism $x$ in $X$. Now, any element $f$ in $E$ can be identified with an element of $C^{\infty}(X)$, the continuous extended real-valued functions (i.e., range $[-\infty, \infty]$ ), each finite on a dense subset of $X$ (technically, the homomorphisms may be identified with valuations to facilitate the mapping). As needed, we will identify elements in $E$ with

their representation in $C^{\infty}(X)$. For a Banach lattice $F$, we identify its universal completion with $C^{\infty}(Y)$, where $Y$ is compact and extremally disconnected (e.g., see [1]). Recall that a Riesz space is said to be universally complete if it is both Dedekind complete and laterally complete (a Riesz space is laterally complete if the supremum of each collection of disjoint positive elements exists).

We will be concerned with several properties of not necessarily linear operators $T$ between Banach lattices $E$ and $F$ and their behavior with respect to positive elements. Throughout this paper, we will assume $T(0)=0$ (if $T$ doesn't take zero to zero, we can consider $T-T(0))$. Since we will be restricting to positive elements $(f \geq 0)$, we will say that $T$ is orthogonally additive if $T(f+g)=T f+T g$ for $f$ and $g$ positive and orthogonal (i.e., $f \wedge g=0$ ), and $T$ is monotone if $T f \leq T g$ whenever $0 \leq f \leq g$. In addition, we will consider the following condition: $T$ will be called subhomogeneous if for each positive scalar $\alpha$, there are positive constants $m(\alpha)$ and $M(\alpha)$ with $m(\alpha)$ unbounded such that for each $f \geq 0$ in $E$,

$$
m(\alpha) T f \leq T(\alpha f) \leq M(\alpha) T(f) .
$$

In general, many of the operators called substitution or Nemystskii operators are orthogonally additive, monotone and subhomogeneous. We recall that an operator $T$ is a substitution operator from one space of real-valued functions on a measure space $X$ to another if for each $f$ in the domain of $T$ and for $x$ in $X$, the image $T f(x)=h(x, f(x))$ where for almost all $x$ the function $h(x$,$) is continuous on the$ reals. Specifically, consider $T$ on $L^{2}$ defined by

$$
T f(x)= \begin{cases}f(x) & \text { if } x \notin f^{-1}([1,2]), \\ 1 & \text { if } x \in f^{-1}([1,2]),\end{cases}
$$

or similar variations, and modifications of $T$ such as $f(x)$ in the top line replaced with an appropriate shift to $f(\varphi(x))$ for a map $\varphi$ from $X$ to $X$. Other examples would include $S$ from $L^{p}$ to $L^{1}$ defined by $S f(x)=f(x)^{p}$. Now the Hammerstein operators associated with these substitution operators, i.e., $L f(y)=$ $\int k(y, x) h(x, f(x)) d \mu$ with positive kernel $k$, are also often orthogonally additive, monotone and subhomogeneous, for example, $A$ from $L^{p}$ to the reals defined by $A f=\int f^{p}$.

As noted above, $T$ (not necessarily linear) from $E$ to $F$ will be called a Carleman operator if the image of the positive elements in the unit ball are order bounded in the universal completion of $F$.

\section{Characterizations of the nonlinear Carleman operator}

We begin with a preliminary lemma on continuity. We note that the unbounded condition for $m(\alpha)$ is needed for the following Lemma. Indeed, $\varphi$ from $\mathbf{R}$ to $\mathbf{R}$ defined by $\varphi(r)=0$ for $r \leq 0$ and $\varphi(r)=r+1$ for $r>0$ fails to be continuous at zero. With the unbounded assumption, we establish the following: 
Lemma 1. If $T$ is an orthogonally additive, monotone, subhomogeneous operator from a Banach lattice $E$ to a normed lattice $F$, then $T$ restricted to a map from the positive elements in $E$ to $F$ is continuous at zero.

Proof. Given $T$ and the function $m$, for any $\varepsilon>0$ and natural number $n$, there is an $\alpha>0$ with the property that $m(\alpha)$ exceeds $n / \varepsilon$ since $m$ is unbounded. Assume $T$ is not continuous. Then there exists an $\varepsilon>0$ so that for each $n$, there is a positive element $g_{n}$ in the ball of radius $1 /\left(n^{2} \alpha\right)$ in $E$ with $\left\|T\left(g_{n}\right)\right\| \geq \varepsilon$. Setting $f_{n}=\alpha g_{n}$, we have $\sum f_{n}$ converges and $T\left(\sum f_{n}\right) \geq T\left(f_{n}\right)$ by the monotone property. Now $T\left(f_{n}\right) \geq m(\alpha) T\left(g_{n}\right)$ and in turn $\left\|T\left(f_{n}\right)\right\| \geq(n / \varepsilon) \varepsilon$, a contradiction.

It may be the case that $T$ is an orthogonally additive, monotone, subhomogeneous operator and $T$ is not continuous everywhere. For example consider $T$ from the reals to itself with $T(r)=r$ for $r \leq 1$ and $T(r)=r+1$ for $r>1$. As a consequence of this Lemma, it is of interest to note that any orthogonally additive, monotone, subhomogeneous operator from $E$ into a space $C(K)$ for $K$ compact is a Carleman operator. Here, $T$ would map $E$ into $C(K)$ with the uniform norm topology and hence would be continuous. Thus the image of the positive elements in the unit ball would be order bounded.

We will say that a function $k$ defined on a subset of $Y \times R \times X$ satisfies the * conditions with respect to measure $\mu$ on $X$, if for $f$ and $g$ in $E$ and each $y$ in a dense subset depending on $f, g$, and $\alpha f$, the function $k(y, f(x), x)$ is measurable on $X$ and satisfies (i) $k(y, f(x), x)=0$ for almost all $x$ in $\{x: f(x)=0\}$ (i.e., $\mu(\{x: k(y, f(x), x) \neq 0\} \cap\{x: f(x)=0\})=0)$, (ii) $k(y, f(x), x) \leq k(y, g(x), x)$ almost everywhere whenever $f \leq g$ and (iii) for each positive $\alpha$, there are positive constants $m(\alpha)$ with $m$ unbounded and $M(\alpha)$ so that $m(\alpha) k(y, f(x), x) \leq$ $k(y, \alpha f, x) \leq M(\alpha) k(y, f(x), x)$ almost everywhere). We now provide a characterization for the Carleman operator when the range space $F$ is complete. Examples of operators satisfying condition (1) or (2) of the following theorem include the composition of substitution operators such as $T$ or $S$ described above with linear Carleman kernels (note, $A$ above is such an operator).

Theorem 1. Let $E$ be a Banach lattice with quasi-interior point, having an order continuous norm, admitting a strictly positive linear functional, and representable in the sense of Schaefer on a compact space $X$. Let $F$ be a Banach lattice with order continuous norm identified with functions on $Y$, where $C^{\infty}(Y)$ corresponds to the universal completion of $F$. For $T$ a continuous map from $E$ to $F$ and $\mu$ the measure associated with the strictly positive functional on $E$ restricted to $C(X)$, the following two conditions are equivalent:

(1) $T$ is an orthogonally additive, monotone, and subhomogeneous Carleman operator.

(2) There is a function $k$ satisfying the * conditions so that for each $f \geq 0$ in $E$,

$$
T f(y)=\int k(y, f(x), x) d \mu
$$

for each $y$ in a dense subset of $Y$ (depending on $f$ ). Further, there exists a dense open subset $O$ of $Y$ so that $T f(y)$ is finite for all $f \geq 0$ in $E$ and all $y$ in $O$.

Proof. We first assume that (1) is satisfied. Then the image of the positive elements in the unit ball are contained in an interval $[0, g]$ and we let $O$ be the set of points in $Y$ where $g$ is finite. We will verify that for $y$ in $O$, there exists a function 
$k(y,$,$) on a subset of R \times X$ corresponding to $T$ via an integral. First we define $v_{y}(A)=(y \circ T)\left(\chi_{A}\right)$ for each set $A$ open and closed in $X$. Recall that $X$ is extremally disconnected since $E$ is Dedekind complete. If there exists a disjoint countable union of sets $\left\{A_{i}\right\}$, each open and closed with union equal to a set $A$ also open and closed, then $f_{n}=\sum_{i=1}^{n} \chi_{A_{i}}$ will have supremum $\chi_{A}$, and by order continuity will converge in norm to $\chi_{A}$. Writing $\chi_{A}$ as $\left(\chi_{A}-f_{n}\right)+f_{n}$, the orthogonal additivity $T$ and the previous lemma (stating $y \circ T$ is continuous at zero) imply that $\sum v_{y}\left(A_{i}\right)=v_{y}(A)$, and thus $v_{y}$ generates a measure on the $\sigma$-algebra generated by the sets both open and closed. Note each function in $E$ is measurable with respect to $v_{y}$ and $v_{y}$ is a Baire measure.

We next verify that $v_{y}$ is absolutely continuous with respect to $\mu$. Given $\mu(A)=$ 0 , let $K$ be a compact $G_{\delta}$ subset of $A$. Choose a decreasing sequence of functions $\left(f_{i}\right)$ with $f_{i} \geq \chi_{K}$ and pointwise infimum $\chi_{K}$. Since $\int f_{i} d \mu$ converges to $\mu(K)$, it follows that $\bigwedge f_{i}=0$. Since the norm is order continuous, $\left\|f_{i}\right\| \rightarrow 0$ and in turn $y \circ T\left(f_{i}\right) \rightarrow 0$ by the continuity of $y \circ T$ at zero. Thus, $v_{y}(K)=0$ and $v_{y}(A)=0$.

Now for each positive real number $\lambda \geq 0$, we define $v_{y}^{\lambda}(A)=(y \circ T)\left(\lambda \chi_{A}\right)$ for the sets which are both open and closed, and extend as above. Arguing as above, $v_{y}^{\lambda} \ll \mu$ and thus we can write $v_{y}^{\lambda}(A)=\int k(y, \lambda, x) \chi_{A} d \mu$ for each open and closed set $A$. We also write $v_{y}^{\lambda}(A)=\int k\left(y, \lambda \chi_{A}, x\right) d \mu$ with the understanding that $k\left(y, \lambda \chi_{A}(x), x\right)=0$ for $x$ not in $A$ and identify $k\left(y, \lambda \chi_{A}+\beta \chi_{B}, x\right)$ with $k\left(y, \lambda \chi_{A}, x\right)+k\left(y, \beta \chi_{B}, x\right)$. Since $T$ is orthogonally additive, if $\left\{A_{i}\right\}$ is a finite collection of pairwise disjoint open and closed sets, then

$$
\begin{gathered}
\int k\left(y, \sum \lambda_{i} \chi_{A_{i}}, x\right) d \mu=\sum \int k\left(y, \lambda_{i} \chi_{A_{i}}, x\right) d \mu \\
=\sum(y \circ T) \lambda_{i} \chi_{A_{i}}=y \circ T\left(\sum \lambda_{i} \chi_{A_{i}}\right) .
\end{gathered}
$$

For $f \geq 0$ in $E$ (as a function on $X$ ), we choose a set of functions $\left\{p_{n}\right\}$ such that $\left\{p_{n}\right\}$ is monotone increasing with supremum $f$ and each $p_{n}$ is of the form $\sum \lambda_{i} \chi_{i}$, for a finite sum where $\chi_{i}$ is the characteristic function of disjoint open and closed sets $A_{i}$. The sets $A_{i}$ may be chosen as the closure of open sets such as $\{x: \alpha<f(x)<\beta\}$ or $\{x: f(x)=\alpha\}^{\circ}$; here, $\lambda_{i}=\alpha$ (the superscript "o" denotes the interior). Now $\left\{p_{n}\right\}$ converges to $f$ in norm since the norm is order continuous.

We will verify that $y \circ T\left(p_{n}\right)$ converges to $y \circ T(f)$ for $y$ in a dense subset $\hat{O}$ of $O$. Although $T$ is continuous, the point evaluation at $y$ is in general not continuous (it is continuous on $C(X)$ with respect to the supremum norm). Since $\left(p_{n}\right)$ converges to $f$, the sequence $\left(T\left(p_{n}\right)\right)$ increases and by continuity of $T$ converges to $T(f)$. For any open set $G$ in $O$, there is a point $x$ with $\left(T f(x)-T p_{m}(x)\right)<1$ for $m$ sufficiently large. Otherwise, $T f-\chi_{K}$ for some open and closed set $K$ in $G$ would be greater than or equal to all $T\left(p_{n}\right)$ and be less than or equal to $T(f)$, contradicting the convergence of $T\left(p_{n}\right)$ to $T(f)$. For subsequent arguments, given a second function $g$, we can choose within $\left\{x:\left(T f(x)-T p_{m}(x)\right)<1\right\}$ a point $\hat{x}$ with $\left(T g(\hat{x})-T q_{n}(\hat{x})\right)<1$ for approximating functions $\left\{q_{n}\right\}$. Thus, $\left(T f(x)-T p_{m}(x)\right)<$ 1 and $\left(T g(x)-T q_{n}(x)\right)<1$ for all $x$ in a compact set $K_{1}$, which is the closure of an open set. Continuing inductively, there exist nested compact sets $K_{n}$ with $\left(T f(x)-T p_{m}(x)\right)<1 / n$ and $\left(T g(x)-T q_{m}(x)\right)<1 / n$ for sufficiently large $m$ and all $x$ in $K_{n}$. It follows that $T\left(p_{n}\right)$ converges pointwise to $T(f)$ for all points in $\bigcap K_{n}$, establishing that $y \circ T\left(p_{n}\right)$ converges to $y \circ T(f)$ for $y$ in a dense subset $\hat{O}$ of $O$ and a corresponding convergence for $g$. 
We next show that $\left\{k\left(y, p_{n}, x\right)\right\}$ is an increasing almost everywhere collection of measurable functions. We first verify that if $A$ is a measurable subset of $X$ with empty interior, then $\mu(A)=0$. For $D$ a compact $G_{\delta}$ subset of $A$, we again choose a decreasing sequence of functions $\left(f_{i}\right)$ with $f_{i} \geq \chi_{D}$ and pointwise infimum $\chi_{D}$. Now $\bigwedge f_{i}$ is less than or equal to $\chi_{D}$ and so $\bigwedge f_{i}$ is equal to zero. Hence, $\left(f_{i}\right)$ converges to zero and, consequently, $\left(\int f_{i} d \mu\right)$ converges to zero. Thus $\mu(D)$ and $\mu(A)$ are equal to zero. Let $Q$ denote the set of points where $k\left(y, p_{n}, x\right)$ is greater than $k\left(y, p_{n+1}, x\right)$ and assume that $K$ denotes a compact $G_{\delta}$ subset of $Q$ with positive measure. Note that $k\left(y, \lambda \chi_{A}, x\right) \chi_{B}=k\left(y, \lambda \chi_{A \cap B}+\lambda \chi_{A \backslash B}, x\right) \chi_{B}=k\left(y, \lambda \chi_{A} \chi_{B}, x\right)$ almost everywhere for open and closed sets $A$ and $B$. It follows that $K^{\circ}$ has positive measure and

$$
\int k\left(y, p_{n}, x\right) \chi_{\overline{K^{\circ}}} d \mu=\int k\left(y, p_{n} \chi_{\overline{K^{\circ}}}, x\right) d \mu>\int k\left(y, p_{n+1} \chi_{\overline{K^{\circ}}}, x\right) d \mu
$$

or

$$
y \circ T\left(p_{n} \chi_{\overline{K^{\circ}}}\right)>y \circ T\left(p_{n+1} \chi_{\overline{K^{\circ}}}\right)
$$

which contradicts the monotonicity of $T$. Thus $K$ and in turn $Q$ have measure zero.

Now we define for each $y, k(y, f, x)$ as the pointwise supremum of $k\left(y, p_{n}(x), x\right)$. It follows from the Monotone Convergence Theorem and the pointwise convergence established above for $\hat{O}$ that $\int k(y, f(x), x) d \mu=y \circ T(f)$ for each $y$ in $\hat{O}$. For the * conditions, (i) follows from the definition of $k$. Note that if condition (ii) were not satisfied for $\hat{O}$, then the monotonicity of $T$ would fail for functions $f \chi_{K}$ and $g \chi_{K}$ with the appropriate choice of $K$. Note that if condition (iii) were not satisfied, then the subhomogeneous condition would fail for some function $f \chi_{K}$. The finiteness condition for the set $O$ is a consequence of the subhomogeneous condition (and that the image of the positive elements in the unit ball are contained in $[0, g]$ ).

For the converse, assume that condition (2) is satisfied. Then $T$ is orthogonally additive, monotone, and subhomogeneous. Now $y \circ T$ for $y$ in $O$ is continuous at zero (by the Lemma). Let $B^{+}$denote the positive elements in the unit ball of $E$ and let $G_{n}$ for each natural number $n$ denote the union of all open sets $U$ such that $T\left(B^{+}\right)(U) \subset[0, n]$. We first verify that $G=\bigcup_{n=1}^{\infty} G_{n}$ is dense in $Y$. If not, there exists an open set $V$ in $O$ disjoint from $G$. Let $E_{n}=\left\{y \in V: T\left(B^{+}\right)(y) \subset[0, n]\right\}$. The continuity of $y \circ T$ at zero and the subhomogeneous condition imply that the union of the sets $E_{n}$ is equal to $V$. Each $E_{n}$ is closed in $V$ as it is an intersection of closed sets $(T f)^{-1}([0, n]) \cap V$. Since $V$ is locally compact, the Baire Category Theorem implies that at least one $E_{n}$ contains an open set which contradicts the fact that $V$ and $G$ are disjoint. Now $G$ is dense and $\overline{G_{m}}$ is open and closed ( $Y$ is extremally disconnected) so that $\chi_{1}=\chi_{\overline{G_{1}}}$, the characteristic function of $\overline{G_{1}}$ and inductively, $\chi_{m}=\chi_{\overline{G_{m}} \backslash \overline{G_{m-1}}}$ for $m>1$ are continuous. Thus, $T\left(B^{+}\right) \subset[0, g]$ for $g=\bigvee_{m} m \chi_{m}$ establishing that $T$ is a Carleman operator. (Note that the value of $g$ on the complement of $\bigcup \bar{G}_{m}$ is infinite since a net from this set convergent to a point in the complement must eventually be outside each fixed $\bar{G}_{m}$ where the value of $g$ is greater than $m$.)

We can provide a characterization similar to Theorem 1 without the continuity assumption on $T$ by substituting a pointwise translation property. We will say that $T$ satisfies the translation property on $Y$ if $\left(T\left(f-g_{n}\right)(y)\right) \rightarrow 0$ for a point $y$ implies that $\left(T\left(g_{n}\right)(y)\right) \rightarrow T(f)(y)$. In Theorem 2 the dense open set is not dependent on the function. 
Theorem 2. Let $E$ be a Banach lattice with quasi-interior point, having an order continuous norm, admitting a strictly positive linear functional, and representable in the sense of Schaefer on $X$, a compact space. Let $F$ be a Banach lattice with order continuous norm identified with functions on $Y$, where $C^{\infty}(Y)$ corresponds to the universal completion of $F$. Let $T$ be a map from $E$ to $F$ which satisfies the translation property on $Y$. For $\mu$ the measure associated with the strictly positive functional on $E$ restricted to $C(X)$, the following two conditions are equivalent:

(1) $T$ is an orthogonally additive, monotone, and subhomogeneous Carleman operator.

(2) There is a function $k$ satisfying the ${ }^{*}$ conditions and an open dense subset $O$ of $Y$ so that for each $y$ in $O$ and $f \geq 0$ in $E$,

$$
T f(y)=\int k(y, f(x), x) d \mu<\infty .
$$

Proof. We first assume that (1) is satisfied. Proceeding as in the proof of Theorem 1 , we again consider the sequence $\left\{p_{n}\right\}$ which converges to $f$. It follows from Lemma 1 that $T\left(f-p_{n}\right)(y) \rightarrow 0$ for each $y$ in $O(O$ is as described in Theorem 1$)$. Now the translation property implies that $\left(y \circ T\left(p_{n}\right)\right)$ converges to $y \circ T(f)$ for all $y$ in $O$ (we need not verify convergence for the subset $\hat{O}$ ). Condition $(2)$ now follows as in the proof of Theorem 1. The proof of (2) implies (1) is again as in the proof of Theorem 1.

In the absence of a measure and without the assumption of either continuity or the translation property, we can establish the following.

Theorem 3. Let $T$ be an orthogonally additive, monotone, subhomogeneous operator from a Banach lattice into a Banach lattice $F$. Let $F$ be identified with $C^{\infty}(Y)$, the universal completion of $F$. The following conditions are equivalent:

(1) $T$ is a Carleman operator.

(2) There exists a dense open subset $O$ of $Y$ with the property that if $f_{n} \geq 0$ and $\left(f_{n}\right)$ converges to zero in $E$, then $\left(T\left(f_{n}\right)(y)\right)$ converges to zero for each $y$ in $O$.

(3) There exists a dense open subset $O$ of $Y$ with the property that $T f(y)$ is finite for each $f \geq 0$ in $E$ and $y$ in $O$.

Proof. Given that (1) is satisfied, Lemma 1 establishes the continuity of $y \circ T$ at zero for $y$ in the set $O$ as in the proof of Theorem 1. For (2) implies (3), the subhomogeneous condition implies that finiteness. Arguing as in the proof of Theorem 1 establishes (3) implies (1).

It would be of interest to understand the behavior of Carleman operators defined without the restriction to positive elements. In the present development the value of the operator on negative elements is arbitrary.

\section{REFERENCES}

1. C. D. Aliprantis and O. Burkinshaw, Positive operators, Academic Press, New York, 1985. MR 87h:47086

2. Sergio Segura De León, Bukhvalov type characterizations of Urysohn operators, Studia Math. 99 (3) (1991), 199-220. MR 92h:47095

3. William Feldman, Carleman operators on Banach lattices, Math. Zeit. 199 (1988), 549-553. MR 90a: 47092

4. J. J. Grobler and P. van Eldik, Carleman operators in Riesz spaces, Indag. Math. 45 (4) (1983), 421-433 also Proc. Kon. Ned. Akad. van Wetensh. A 86 (4) (1983). MR 85k:47069 
5. J. M. Mazón and S. Segura De León, Order bounded orthogonally additive operators, Rev. Roumaine Math. Pures Appl. 35 (1990), 329-353. MR 92b:47087

6. H. H. Schaefer, Banach lattices and positive operators, Springer, Berlin, 1974. MR 54:11023

Department of Mathematical Sciences, University of Arkansas, Fayetteville, ARKANSAS 72701

E-mail address: wfeldman@comp.uark.edu 